\title{
Sequential and parallel schemes for adaptive 2-D parameter estimation with application to image estimation
}

\author{
U B DESAI, Z D DITTIA, P S KUMAR and P Y MUNDKUR \\ Department of Electrical Engineering, Indian Institute of Technology, \\ Powai, Bombay 400076, India
}

\begin{abstract}
In this paper, the non-causal quarter plane 2-D Recursive Least Squares (2D-RLS) algorithm for adaptive processing is developed. The complexity of this algorithm turns out to be $O\left(L^{6}\right)$ per iteration, for an $L \times L$ window. With the aim of reducing this complexity, the matrix gains appearing in the algorithm are replaced by scalar gains. This approach yields the Approximate 2-D Recursive Least Squares (A2D-RLS) algorithm, which is shown to have a complexity of $O\left(L^{2}\right)$. With the objective of reducing the computation time even further, a parallel scheme is developed for the A2D-RLS algorithm. Since the algorithm is inherently sequential, its parallelization involves some more approximations. The desired accuracy of the estimated parameters is shown to place an upper bound on the number of processors. The parallel scheme is suitable for implementation on shared memory as well as distributed memory machines. The algorithm is applied to the problem of image estimation. Simulation results giving speed-up, efficiency, and the accuracy of the estimated image are presented.
\end{abstract}

Keywords. Adaptive 2-D parameter estimation; image estimation; adaptive processing; recursive least squares algorithm.

\section{Introduction}

Adaptive processing techniques have found widespread use in signal processing, image processing and communication problems (Widrow \& Stearns 1985; Haykin 1986). Most of the work in the area of adaptive processing has been on processing one-dimensional (1-D) signals. Of late, there has been a growing interest in the adaptive processing of two-dimensional (2-D) signals. In this context, recent developments include the 2-D Least Mean Squares (2D-LMS) algorithm (Hadhoud \& Thomas 1988) and a fast version of the 2-D Recursive Least-Squares (F2D-RLS) algorithm (Boutalis et al 1989). Other papers in this area include Chan \& Lim (1985) and Tekalp et al (1986).

In this paper, we first develop the 2D-RLS algorithm using a non-causal quarter plane as the underlying model. This algorithm has a very high computational complexity $-O\left(L^{6}\right)$ per iteration for an $L \times L$ support window. To obtain a computationally viable algorithm, we propose an approximation scheme where the time varying matrix adaptation parameters are replaced by time varying scalar 
adaptation parameters. The algorithm so obtained has a computational complexity of $O\left(L^{2}\right)$ per iteration, and shall henceforth be referred to as the Approximate 2-D Recursive Least Squares (A2D-RLS) algorithm. In order to achieve further speed-up, a parallel implementation of the A2D-RLS algorithm is obtained. Parallelization requires certain approximations and a few tricks. The latter part of the paper deals with this parallel algorithm.

Remark 1: We would like to remark that both the 2D-RLS and the A2D-RLS algorithms exploit a parallel method for solving a system of equations involving a doubly block approach to 2-D parameter estimation. Hence, we have concentrated on the parallel implementation of the A2D-RLS algorithm, with the understanding that our parallelization schemes can easily be extended to the 2D-RLS algorithm.

Remark 2: Recently Kalouptsidis \& Theodoridis (1989) have developed a parallel algorithm for noncausal 2-D Wiener filters with symmetrical impulse response. They exploit a parallel method for solving a system of equations involving a doubly block Toeplitz matrix. Moreover, they assume that the covariance lags of the underlying processes are available. We neither assume the symmetry of the impulse response nor the availability of the covariance lags. Our parallel implementation is adaptive, in the sense that it directly operates on the data.

Simulations of the parallel A2D-RLS algorithm were carried out on a synthetic AR image using ASTRO, a parallel computer simulator and performance monitor (Dittia \& Desai 1989). The simulation results obtained include plots of the efficiency and speedup for varying numbers of processors, and are reported towards the end of the paper. Simulations were also carried out for estimating a noisy Girl image using A2D-RLS, 2D-LMS, and F2D-RLS algorithms. Comparative results are reported in $\$ 4$.

We would like to remark that the sequential 2D-RLS and A2D-RLS algorithms (tables 1 and 2) were first reported in Desai \& Kumar (1989).

Table 1. The 2D-RLS algorithm.

\begin{tabular}{lc}
\hline Initialization & for $m \geqslant(L+1) / 2$ and $n \geqslant(L+1) / 2$ \\
$Q_{m+1}((L+1) / 2)$ & $=\delta_{q}^{-1} I, \delta_{q}=$ a small positive constant \\
$S_{n+1}((L+1) / 2)$ & $=\delta_{s}^{-1} I, \delta_{s}=$ a small positive constant \\
$x_{m+1}((L+1) / 2)$ & $=0, \beta_{n+1}((L+1) / 2)=0$ \\
$\hat{O}((L+1) / 2,(L+1) / 2)=$ & 0 \\
$P((L+1) / 2,(L+1) / 2)=$ & $\rho^{-1} I, \rho=a$ small positive constant \\
Vertical update & \\
$Q_{m+1}(n)$ & $\quad Q_{m+1}(n-1)-Q_{m+1}(n-1) Y(m+1, n) \times$ \\
& $\quad \times Y^{T}(m+1, n) Y_{m+1}(n-1)$
\end{tabular}


Table 1. (Continued)

\begin{tabular}{|c|c|}
\hline$x_{m+1}(n)$ & $\begin{aligned}=x_{m+1} & (n-1)+Q_{m+1}(n) Y(m+1, n) \times \\
& \times\left[y(m+1, n)-Y^{T}(m+1, n) \alpha_{m+1}(n-1)\right]\end{aligned}$ \\
\hline$\hat{\theta}(m+1, n)$ & $\begin{array}{l}=Q_{m+1}(n)\left[P(m, n)+Q_{m+1}(n)\right]^{-1} \hat{\theta}(m, n)+ \\
\quad+P(m, n)\left[P(m, n)+Q_{m+1}(n)\right]^{-1} \alpha_{m+1}(n)\end{array}$ \\
\hline$P(m+1, n)$ & $=Q_{m+1}(n)\left[P(m, n)+Q_{m+1}(n)\right]^{-1} P(m, n)$ \\
\hline \multicolumn{2}{|c|}{ Horizontal update } \\
\hline$S_{n+1}(m+1)$ & $\begin{aligned}=S_{n+1}(m)-S_{n+1}(m) Y(m+1, n+1) \times \\
\quad \times\left[1+Y^{T}(m+1, n+1) S_{n+1}(m) Y(m+1, n+1)\right]^{-1} \times \\
\quad \times Y^{T}(m+1, n+1) S_{n+1}(m)\end{aligned}$ \\
\hline$\beta_{n+1}(m+1)$ & $\begin{aligned}=\beta_{n+1}(m)+S_{n+1}(m+1) Y(m+1, n+1) \times \\
\quad \times\left[y(m+1, n+1)-Y^{T}(m+1, n+1) \beta_{n+1}(m)\right]\end{aligned}$ \\
\hline$\hat{\theta}(m+1, n+1)$ & $\begin{aligned} &=S_{n+1}(m+1)\left[P(m+1, n)+S_{n+1}(m+1)\right]^{-1} \hat{\theta}(m+1, n)+ \\
&+P(m+1, n)\left[P(m+1, n)+S_{n+1}(m+1)\right]^{-1} \beta_{n+1}(m+1)\end{aligned}$ \\
\hline$P(m+1, n+1)$ & $=S_{n+1}(m+1)\left[P(m+1, n)+S_{n+1}(m+1)\right]^{-1} P(m+1, n)$ \\
\hline
\end{tabular}

Table 2. The approximate 2D-RLS algorithm - preliminary version.

$$
\begin{aligned}
& \text { Initialization } \\
& \text { for } m \geqslant(L+1) / 2 \text { and } n \geqslant(L+1) / 2 \\
& q_{m+1}^{-1}((L+1) / 2)=\delta_{q}, \delta_{q}=\text { a small positive constant } \\
& s_{n+1}^{-1}((L+1) / 2) \quad=\delta_{s}, \delta_{s}=\text { a small positive constant } \\
& \alpha_{m+1}((L+1) / 2) \quad=0, \beta_{n+1}((L+1) / 2)=0 \\
& \hat{\theta}((L+1) / 2,(L+1) / 2)=0 \\
& \rho^{-1}((L+1) / 2,(L+1) / 2)=\rho, \rho=a \text { small positive constant }
\end{aligned}
$$

\section{Vertical update}

$$
\begin{aligned}
& q_{m+1}^{-1}(n) \quad=q_{m+1}^{-1}(n-1)+Y^{T}(m+1, n) Y(m+1, n) \\
& x_{m+1}(n) \quad=\alpha_{m+1}(n-1)+q_{m+1}(n) Y(m+1, n) \times \\
& \times\left[y(m+1, n)-Y^{T}(m+1, n) \alpha_{m+1}(n-1)\right] \\
& \hat{\theta}(m+1, n) \\
& =\left[q_{m+1}(n) \hat{\theta}(m, n)+p(m, n) \alpha_{m+1}(n)\right] /\left[p(m, n)+q_{m+1}(n)\right] \\
& p^{-1}(m+1, n) \\
& =p^{-1}(m, n)+q_{m+1}^{-1}(n)
\end{aligned}
$$

Horizontal update

$$
\begin{aligned}
& s_{n+1}^{-1}(m+1) \\
& =s_{n+1}^{-1}(m)+Y^{T}(m+1, n+1) Y(m+1, n+1) \\
& \beta_{n+1}(m+1) \\
& =\beta_{n+1}(m)+s_{n+1}(m+1) Y(m+1, n+1) \times \\
& \times\left[y(m+1, n+1)-Y^{T}(m+1, n+1) \beta_{n+1}(m)\right] \\
& \hat{\theta}(m+1, n+1) \\
& =\left[s_{n+1}(m+1) \hat{\theta}(m+1, n)+p(m+1, n) \beta_{n+1}(m+1)\right] \div \\
& \div\left[p(m+1, n)+s_{n+1}(m+1)\right] \\
& p^{-1}(m+1, n+1) \\
& =p^{-1}(m+1, n)+s_{n+1}^{-1}(m+1)
\end{aligned}
$$




\section{The 2D-RLS and A2D-RLS algorithms}

A two-dimensional data set can be looked upon as a 2-D array of real numbers. Since such data sets are predominantly encountered in image processing, we shall in future refer to a 2-D data set (or a 2-D signal) as an image, with the understanding that our work is applicable not just in image processing, but also in other areas involving processing of 2-D data.

In figure 1 , we have shown an image and a $3 \times 3$ non-causal window about the point $(2,2)$ of the image. Although our algorithm is applicable to both causal and non-causal models ${ }^{\dagger}$, we have chosen to concentrate on the latter in this report. In general, consider an image and an $L \times L$ non-causal window $\mathscr{R}$ centred at the point $(m, n)$ of the image. Let $y(m, n)$ be the two dimensional signal associated with this image. We assume that $y(m, n)$ obeys the AR model

$$
y(m, n)=\sum_{(i, j) \in\{,(i, j) \neq(m, n)} a(i, j) y(m-i, n-j)+v(m, n)
$$

where $v(\cdot, \cdot)$ is a sequence of independent random variables with zero mean. These variables can be looked upon as the noise term in the equation. Our objective is to estimate the parameters $a(\cdot$,$) in this model. To this end, we define the L^{2}-1$ dimensional vecters as in chart 1 where null designates the fact that the corresponding element is not taken as belonging to the vector. With these definitions, the equation for the model becomes

$$
y(m, n)=\theta^{T} Y(m, n)+v(m, n) .
$$

With the ultimate aim of recursively estimating $\theta$, we seek to minimize the squared error

$$
E=\sum_{i=1}^{m} \sum_{j=1}^{n}\left[y(i, j)-\theta^{T} Y(i, j)\right]^{2} .
$$

Let $\hat{\theta}(m, n)$ denote the solution to this minimization problem. Then it is easily shown that the nonrecursive solution is obtained by solving the normal equation

$$
\left[\sum_{i=1}^{m} \sum_{j=1}^{n} Y(i, j) Y^{T}(i, j)\right] \hat{\theta}(m, n)=\sum_{i=1}^{m} \sum_{j=1}^{n} y(i, j) Y(i, j) \text {. }
$$

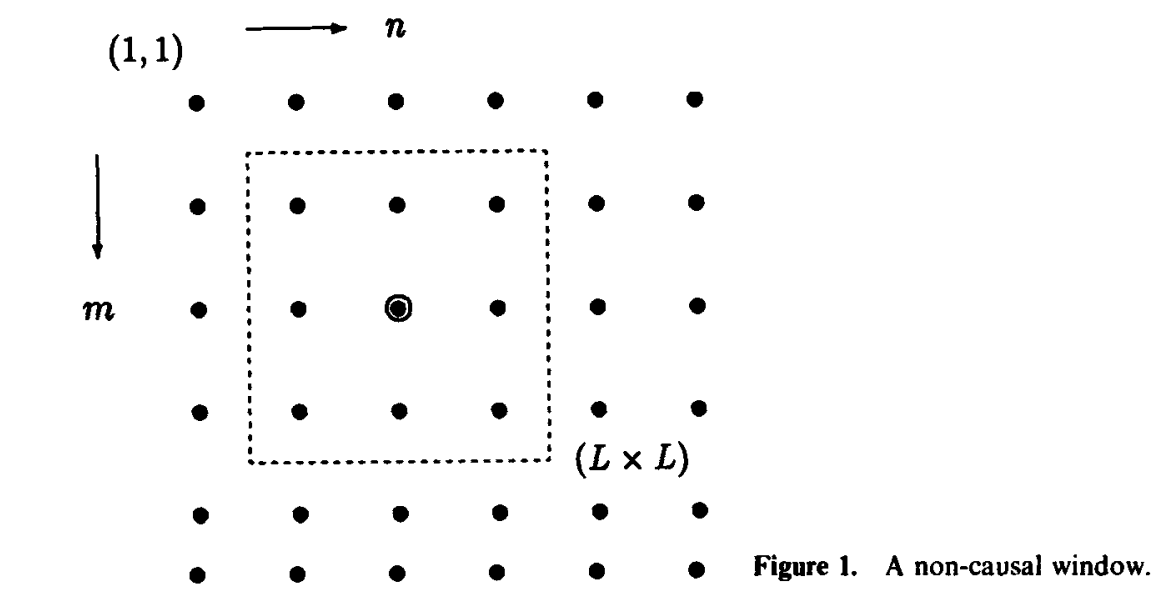

\footnotetext{
${ }^{+}$In fact, the non-causal model is often preferred in image processing.
} 


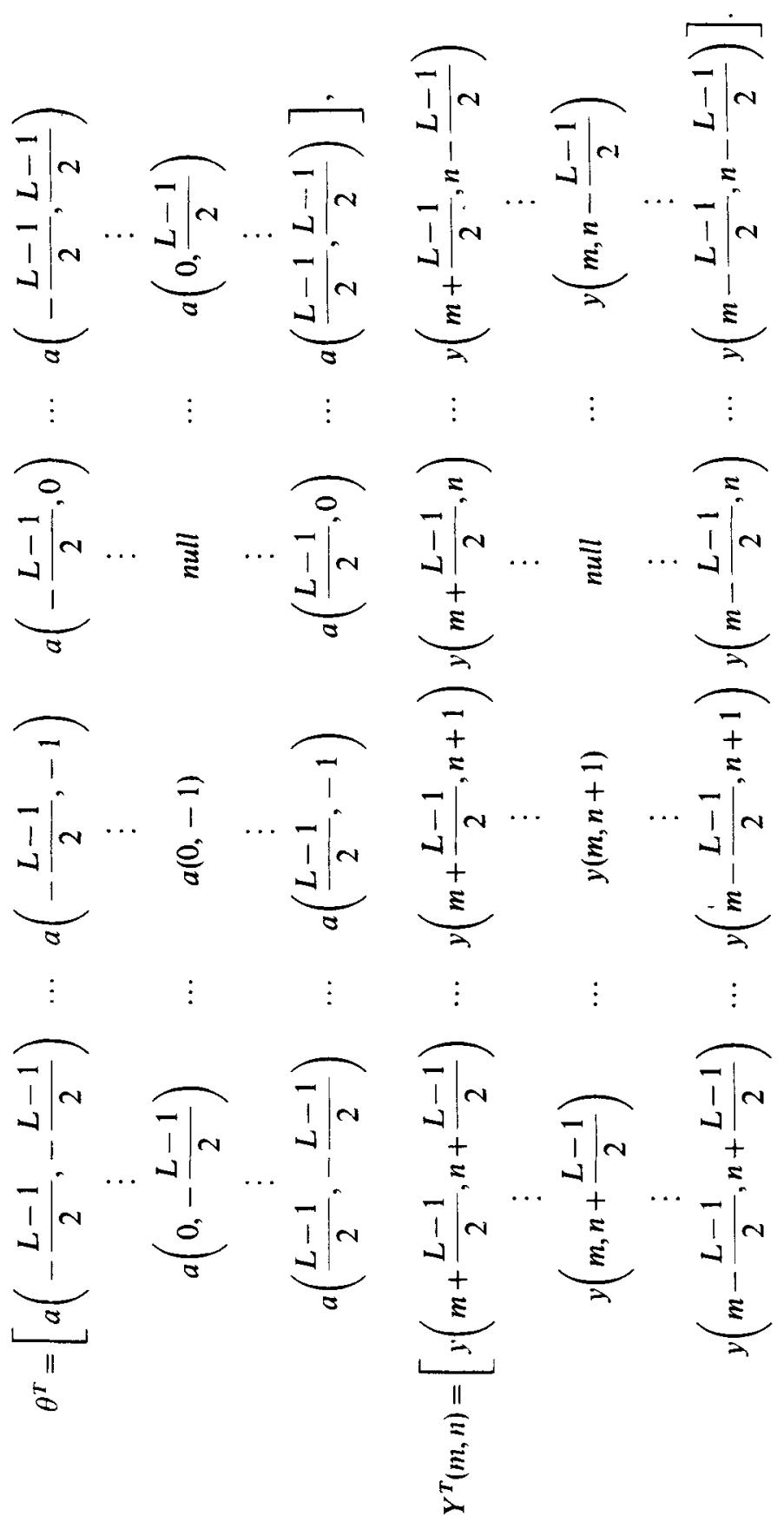

$\frac{\dot{2}}{\text { 농 }}$

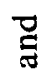


Our aim is to express this equation in a recursive form. To this end, we express $\hat{\theta}(m+1, n)$ in terms of $\hat{\theta}(m, n)$. We have

$$
\begin{aligned}
\hat{\theta}(m+1, n)= & {\left[\sum_{i=1}^{m+1} \sum_{j=1}^{n} Y(i, j) Y^{T}(i, j)\right]^{-1}\left[\sum_{i=1}^{m+1} \sum_{j=1}^{n} y(i, j) Y(i, j)\right] } \\
= & {\left[\sum_{i=1}^{m} \sum_{j=1}^{n} Y(i, j) Y^{T}(i, j)+\sum_{j=1}^{n} Y(m+1, j) Y^{T}(m+1, j)\right]^{-1} } \\
& \times\left[\sum_{i=1}^{m} \sum_{j=1}^{n} y(i, j) Y(i, j)+\sum_{j=1}^{n} y(m+1, j) Y(m+1, j)\right] .
\end{aligned}
$$

If we define

$$
\begin{aligned}
& P(m, n)=\left[\sum_{i=1}^{m} \sum_{j=1}^{n} Y(i, j) Y^{T}(i, j)\right]^{-1} \\
& Q_{m+1}(n)=\left[\sum_{j=1}^{n} Y(m+1, j) Y^{T}(m+1, j)\right]^{-1},
\end{aligned}
$$

then we can write

$$
\begin{aligned}
\hat{\theta}(m+1, n)= & {\left[P^{-1}(m, n)+Q_{m+1}^{-1}(n)\right]^{-1} \times } \\
& \times\left[\sum_{j=1}^{n} y(m+1, j) Y(m+1, j)+\sum_{i=1}^{m} \sum_{j=1}^{n} y(i, j) Y(i, j)\right] .
\end{aligned}
$$

In the above we are assuming the existence of the necessary inverses. Using the above definitions, we get

$$
\hat{\theta}(m, n)=P(m, n)\left[\sum_{i=1}^{m} \sum_{j=1}^{n} Y(i, j) Y^{T}(i, j)\right]
$$

We now list two possible expansions of $P(m+1, n)$ and use them in (4) to obtain another expression for $\hat{\theta}(m+1, n)$ :

where

$$
\begin{aligned}
P(m+1, n)= & Q_{m+1}(n)\left[P(m, n)+Q_{m+1}(n)\right]^{-1} P(m, n) \\
= & P(m, n)\left[P(m, n)+Q_{m+1}(n)\right]^{-1} Q_{m+1}(n), \\
\hat{\theta}(m+1, n)= & Q_{m+1}(n)\left[P(m, n)+Q_{m+1}(n)\right]^{-1} \hat{\theta}(m, n)+ \\
& \quad+P(m, n)\left[P(m, n)+Q_{m+1}(n)\right]^{-1} \alpha_{m+1}(n),
\end{aligned}
$$

$$
\alpha_{m+1}(n)=Q_{m+1}(n) \sum_{j=1}^{n} y(m+1, j) Y(m+1, j)
$$

To obtain $\alpha_{m+1}(n)$, we shall use recursion along the $(m+1)$ th row. Thus, we have to express $\alpha_{m+1}(n)$ in terms of $\alpha_{m+1}(n-1)$. We have

$$
\alpha_{m+1}(n-1)=Q_{m+1}(n-1) \sum_{j=1}^{n-1} y(m+1, j) Y(m+1, j),
$$


and

$$
\begin{aligned}
Q_{m+1}(n) & =\left[\sum_{j=1}^{n} Y(m+1, j) Y^{T}(m+1, j)\right]^{-1} \\
& =\left[Q_{m+1}^{-1}(n-1)+Y(m+1, n) Y^{T}(m+1, n)\right]^{-1}
\end{aligned}
$$

Using the matrix inversion formula

we get

$$
\left(A+u v^{T}\right)^{-1}=A^{-1}-\left(A^{-1} u v^{T} A^{-1}\right) /\left(1+v^{T} A^{-1} u\right)
$$

$$
\begin{aligned}
Q_{m+1}(n)= & Q_{m+1}(n-1)- \\
& -\frac{Q_{m+1}(n-1) Y(m+1, n) Y^{T}(m+1, n) Q_{m+1}(n-1)}{1+Y^{T}(m+1, n) Q_{m+1}(n-1) Y(m+1, n)},
\end{aligned}
$$

and so

$$
\begin{aligned}
\alpha_{m+1}(n)= & \alpha_{m+1}(n-1)+Q_{m+1}(n) Y(m+1, n) y(m+1, n)- \\
& -\frac{Q_{m+1}(n-1) Y(m+1, n) Y^{T}(m+1, n) \alpha_{m+1}(n-1)}{1+Y^{T}(m+1, n) Q_{m+1}(n-1) Y(m+1, n)} .
\end{aligned}
$$

With a view to simplifying this update equation further, we use the result of (7) to get

$$
\begin{aligned}
& Q_{m+1}(n) Y(m+1, n)=Q_{m+1}(n-1) Y(m+1, n)- \\
&-\left\{\begin{array}{c}
{\left[Q_{m+1}(n-1) Y(m+1, n) Y^{T}(m+1, n) Q_{m+1}(n-1) \times\right.} \\
\times Y(m+1, n)]
\end{array}\right\} \\
&= Q_{m+1}(n-1) Y(m+1, n) \times \\
& \times\left[1-\frac{Y^{T}(m+1, n) Q_{m+1}(n-1) Y(m+1, n)}{1+Y^{T}(m+1, n) Q_{m+1}(n-1) Y(m+1, n)}\right]
\end{aligned}
$$

Recognizing the term in square brackets as an expansion obtained using the matrix inversion formula, we can go backwards and replace this term by its unexpanded version, to get

$$
\begin{aligned}
Q_{m+1}(n) Y(m+1, n)=Q_{m+1} & (n-1) Y(m+1, n) \times \\
& \times\left[1+Y^{T}(m+1, n) Q_{m+1}(n-1) Y(m+1, n)\right]^{-1} .
\end{aligned}
$$

Using this in the update equation for $\alpha_{m+1}(n)$, we obtain

$$
\begin{aligned}
\alpha_{m+1}(n)=\alpha_{m+1}(n-1)+Q_{m+1}(n) Y(m+1, n) \times \\
\quad \times\left[y(m+1, n)-Y^{T}(m+1, n) \alpha_{m+1}(n-1)\right] .
\end{aligned}
$$

The updating equations along the horizontal direction can be analogously derived. We list these equations along with appropriate initialization in table 1 . In this table, the definition for $S_{n+1}(m+1)$ is

$$
S_{n+1}(m+1)=\sum_{i=1}^{m+1} Y(i, n+1) Y^{T}(i, n+1) \text {. }
$$


Note that, this matrix adaption parameter is the counterpart of $Q_{m+1}(n)$ in the vertical update. Similarly, $\beta$ is the vector in the horizontal update corresponding to $\alpha$ in the vertical update.

It is easily verified that the algorithm in table 1 has a complexity of $O\left(L^{6}\right)$ flops per iteration, the primary computational burden coming from the inversion of the $\left(L^{2}-1\right) \times\left(L^{2}-1\right)$ matrices $P(m, n)+Q_{m+1}(n)$ and $P(m+1, n)+S_{n+1}(m+1)$. To overcome this, the following approximations (involving the replacement of the time-varying matrix gains $(Q, S, P)$ by time-varying scalar gains $(q, s, p))$ are made:

$$
\begin{aligned}
q_{m+1}^{-1}(n) & =\operatorname{trace}\left[Q_{m+1}^{-1}(n)\right], \\
s_{n+1}^{-1}(m+1) & =\operatorname{trace}\left[S_{n+1}^{-1}(m+1)\right], \\
p^{-1}(m, n) & =\operatorname{trace}\left[P^{-1}(m, n)\right] .
\end{aligned}
$$

Note that the trace has been used to embody the essence of the matrix adaptation parameters, and also to obtain simple updating equations.

It is easily established that

$$
\begin{aligned}
q_{m+1}^{-1}(n) & =\sum_{j=1}^{n} \operatorname{trace}\left[Y(m+1, j) Y^{T}(m+1, j)\right] \\
& =\sum_{j=1}^{n} Y^{T}(m+1, j) Y(m+1, j),
\end{aligned}
$$

which gives the updating equation for $q_{m+1}^{-1}(n)$ as

$$
q_{m+1}^{-1}(n)=q_{m+1}^{-1}(n-1)+Y^{T}(m+1, n) Y(m+1, n) .
$$

Following an analogous technique yields the update equation for $s_{n+1}^{-1}(m+1)$ as

$$
s_{n+1}^{-1}(m+1)=s_{n+1}^{-1}(m)+Y^{T}(m+1, n+1) Y(m+1, n+1) .
$$

As concerns the scalar adaptation parameter $p^{-1}(\cdot, \cdot)$, it is not difficult to show that the corresponding update equations are

$$
\begin{aligned}
& p^{-1}(m+1, n)=p^{-1}(m, n)+q_{m+1}^{-1}(n), \\
& p^{-1}(m+1, n+1)=p^{-1}(m+1, n)+s_{n+1}^{-1}(m+1) .
\end{aligned}
$$

The above approximations lead to a raw form of the A2D-RLS algorithm, the update equations for which are listed in table 2 . Some refinements are in order before this algorithm can be used. These refinements involve further approximations, which we describe in the next few paragraphs.

First, note that we had initialized $\alpha$ and $\beta$ to zero vectors in table 2 , since nothing is known about them. A lơk at the update equations for $\hat{\theta}$ tells us that as the algorithm approaches convergence, $\alpha$ and $\beta$ must both approach $\hat{\theta}$. Therefore, it is conceivable that using the most recent value of $\hat{\theta}$ as the initial value of $\alpha$ or $\beta$ would improve the rate of convergence, and we have found that this is indeed true in practice. This initialization forms an important component of the modified algorithm, and is responsible for a significant increase in its convergence rate.

Although a one-dimensional algorithm derived using the above techniques would work as desired, its two-dimensional counterpart requires what we shall call 
re-initialization, in order to converge to the desired solution. The additional requirement stems from the fact that while the non-causal (or causal) window contains only $L$ data points in the one-dimensional case, its two-dimensional counterpart contains many more data points $-\left(L^{2}-1\right)$ to be precise. Hence, we see that each update of the scalars $q^{-1}$ and $s^{-1}$ involves addition of the squares of $\left(L^{2}-1\right)$ data values to its previous value. Therefore, as the algorithm proceeds, $q$ and $s$ become smaller and smaller, and so does the value of the scalar $p$. One look at the update equation for $\hat{\theta}$ tells us that a very small $p$ implies a negligible change in $\hat{\theta}$ i.e., there is hardly any adaptation. To curb this trend, it is important to limit the increase in the values of $p^{-1}, q^{-1}$ and $s^{-1}$. With this in mind, we incorporate two changes in the A2D-RLS algorithm. The first involves a reduction in the contribution of the $Y^{T} Y$ term to the increase in $q^{-1}$ and $s^{-1}$ by multiplication by the normalizing factor $1 /\left(L^{2}-1\right)$.

The second modification places a check on uncontrolled increase in the value of $p^{-1}$ by placing an upper bound on this scalar. After each update of $\hat{\theta}$ and $p$, the value of $p$ is compared to a threshold, and the moment it drops below this threshold, its value is re-initialized to its value at the start of the program. One could select a fixed value for the threshold and then proceed with the algorithm. We found that an alternate approach works better; it involves comparing the relative change in the vector $\hat{\theta}$. We reinitialize $p^{-1}$ if $\left\|\hat{\theta}_{\text {new }}-\hat{\theta}_{\text {old }}\right\| /\left\|\hat{\theta}_{\text {old }}\right\| \leqslant$ some constant. If this constant selected is 0.01 , the re-initialization occurs when the relative change in $\hat{\theta}$ is less than $1 \%$. Re-initialization was shown to introduce a significant improvement in the algorithm's rate of convergence. The final sequential version of the A2D-RLS algorithm is presented in table 3.

\section{Parallelism in the algorithm}

Before we begin the discussion on developing a parallel scheme for the A2D-RLS algorithm, we would like to mention a few relevant references. With regard to parallel computers and their applications, a good reference is Fox et al (1988). Moreover, an interesting approach to analysis of parallelism in algorithms can be found in Lord et al (1980) and Kumar \& Kowalik (1986).

The computational flow diagram for the A2D-RLS algorithm is shown in figure 2, where we have assumed a $6 \times 6$ image and a $3 \times 3$ non-causal window. Circled pixels indicate the point about which the non-causal window is constructed, at each iteration.

Iterations proceed along the full arrows forming a staircase along the diagonal of the image. Each iteration involves the update of $\hat{\theta}$ and $p^{-1}$. We have chosen to represent a vertical update by a task symbol $V$, and a horizontal update by $H$. In what follows, we adopt the convention that superscripts indicate row numbers and subscripts indicate column numbers for the data matrix of the 2-D system. Thus, we designate the task represented by a full vertical arrow in column $i$ of the image by the symbol $V_{i}$. Similarly, $H^{i}$ symbolizes the task represented by a full horizontal arrow in row $i$ of the image. These task symbol assignments are shown in figure 2.

Figure 2 also shows a number of dashed horizontal and vertical arrows. These represent the update of $\alpha$ and $q^{-1}$, or $\beta$ and $s^{-1}$ from one pixel to the next. Again, for convenience, we shall represent these tasks by appropriate symbols, as shown in 
Table 3. The approximate 2D-RLS algorithm - final version.

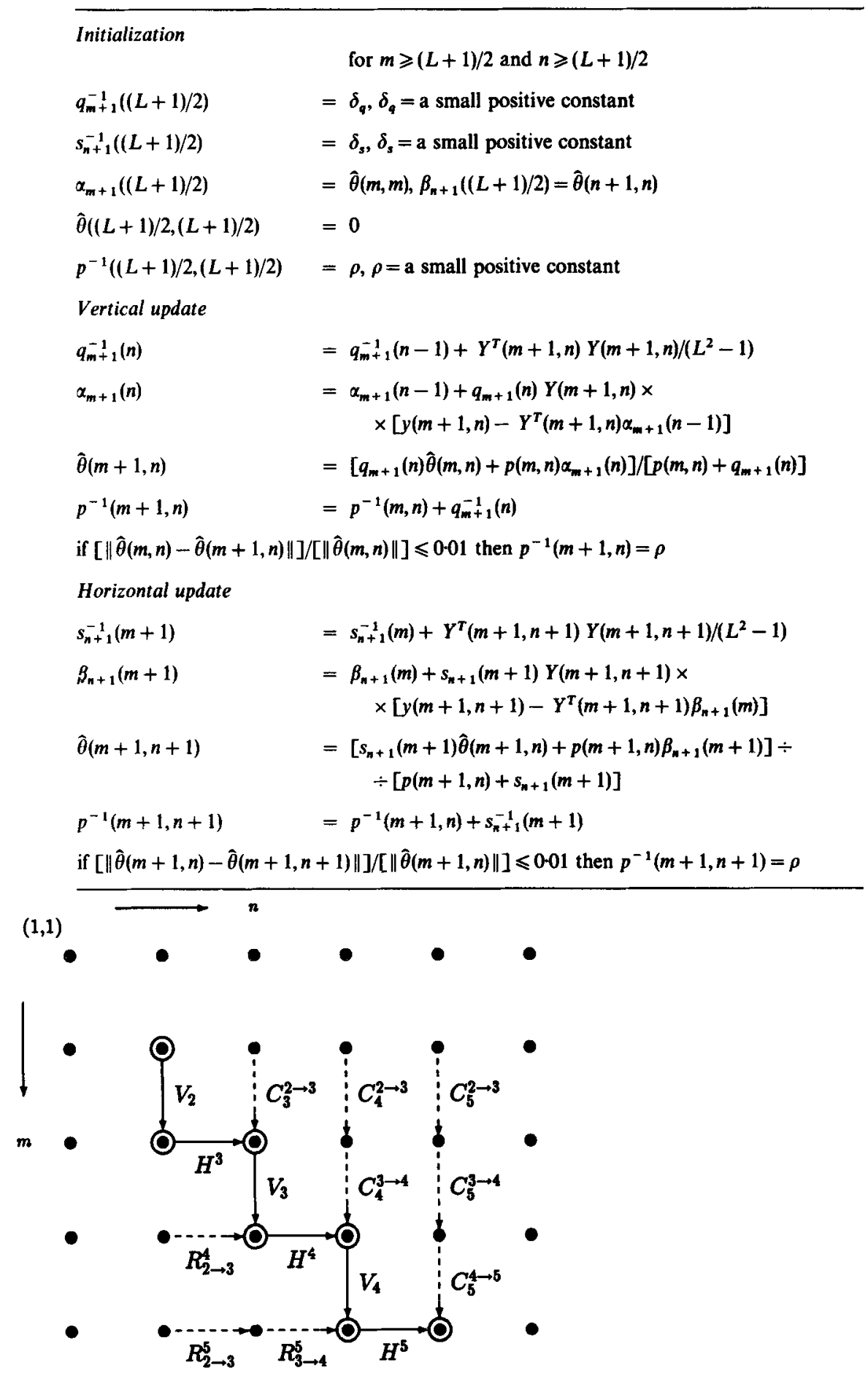

Figure 2. Computational flow dia(6,6) gram. 
Table 4. Task assignments.

\begin{tabular}{llc}
\hline $\begin{array}{l}\text { Task } \\
\text { symbol }\end{array}$ & \multicolumn{1}{c}{$\begin{array}{c}\text { Update } \\
\text { equations }\end{array}$} & $\begin{array}{c}\text { Computational } \\
\text { complexity }\end{array}$ \\
\hline$V_{j}$ & $\hat{\theta}(j+1, j)=\left[q_{j+1}(j) \hat{\theta}(j, j)+p(j, j) \alpha_{j+1}(j)\right] /\left[p(j, j)+q_{j+1}(j)\right]$ & \\
& $p^{-1}(j+1, j)=p^{-1}(j, j)+q_{j+1}^{-1}(j)$ & $O\left(2 L^{2}\right)$ \\
$H^{i}$ & $\hat{\theta}(i, i)=\left[s_{i}(i) \hat{\theta}(i, i-1)+p(i, i-1) \beta_{i}(i)\right] /\left[p(i, i-1)+s_{i}(i)\right]$ & \\
& $p^{-1}(i, i)=p^{-1}(i, i-1)+s_{i}^{-1}(i)$ & $O\left(2 L^{2}\right)$ \\
$R_{j \rightarrow j+1}^{i}$ & $q_{j}^{-1}(j+1)=q_{i}^{-1}(j)+Y^{T}(i, j+1) Y(i, j+1)$ & \\
& $\alpha_{i}(j+1)=\alpha_{i}(j)+q_{i}(j+1) Y(i, j+1)\left[y(i, j+1)-Y^{T}(i, j+1) \alpha_{i}(j)\right]$ & $O\left(3 L^{2}\right)$ \\
$C_{j}^{i \rightarrow i+1}$ & $s_{j}^{-1}(i+1)=s_{j}^{-1}(i)+Y^{T}(i+1, j) Y(i+1, j)$ & \\
\hline & $\beta_{j}(i+1)=\beta_{j}(i)+s_{j}(i+1) Y(i+1, j)\left[y(i+1, j)-Y^{T}(i+1, j) \beta_{j}(i)\right]$ & $O\left(3 L^{2}\right)$ \\
\hline
\end{tabular}

figure 2. $R$ will represent the row update of $\alpha$ and $q^{-1}$, while $C$ will represent the column update of $\beta$ and $s^{-1}$. Thus, $R_{j \rightarrow j+1}^{i}$ will designate the task represented by a dashed horizontal arrow in row $i$, stretching from the pixel in column $j$ to that in column $j+1$ (in other words, the arrow extends from the point $(i, j)$ to $(i, j+1)$ ). Similarly, $C_{j}^{i \rightarrow i+1}$ designates the task represented by a dashed vertical arrow in column $j$, having its tail in row $i$ and its head in row $i+1$ (i.e., the arrow extends from point $(i, j)$ to $(i+1, j))$.

For brevity, we shall often call a group of tasks using the symbols they are represented by; thus, for example, we may refer to the $H$-tasks or the $R$-tasks.

The first two columns of table 4 list the task symbol and the corresponding update equations. Noting that $\hat{\theta}, \alpha$, and $\beta$ are $L^{2}-1$ dimensional vectors, while $p, q$ and $s$ are scalars, we can calculate the computational complexity of the update equations. These are listed in the third column of table 4. We note from this table that although all the tasks have $O\left(L^{2}\right)$ computational complexity, the $R$ and $C$ tasks involve $50 \%$ more computations than the $V$ and $H$ tasks. However, in our parallel implementation, we assume that a processor doing a $V$ or $H$ task takes as much time to do this task as a processor doing an $R$ or $C$ task would. As we shall see later, this assumption does not seriously degrade performance. Armed with this supposition, we set out to analyse the parallelism in the algorithm.

For the moment, we assume that the initialization of $\alpha$ or $\beta$ to the latest computed value of $\theta$ is not required; i.e., we assume that the algorithm proceeds as shown in table 2. Later, we shall include the above initialization, and see how the resulting loss in parallelism can be bartered for a degradation in convergence while retaining the same degree of parallelism.

From the equations listed in table 3, we note that $H^{3}$ requires the results of $V_{2}$ and $C_{3}^{2 \rightarrow 3}$. Similarly, $V_{3}$ requires the results of $H^{3}$ and $R_{2}^{4} \rightarrow 3 . H^{4}$ requires the results of $V_{3}$ and $C_{4}^{3 \rightarrow 4}$, which in turn requires the results of $C_{4}^{2 \rightarrow 3}$, and so on. These relationships for a general task system are given in table 5 . These precedence constraints facilitate the construction of a "maximally parallel task system". By this, we mean that at any time instant, we execute as many tasks in parallel as possible, subject to the precedence constraints. Such a maximally parallel task system for the example in figure 2 is shown in figure 3. 
Table 5. Precedence constraints.

\begin{tabular}{lc}
\hline Task & Requires results from \\
\hline$V_{j}$ & $H^{j}, R_{j-1}^{j+1}+j$ \\
$H^{i}$ & $V_{i-1}, C_{i}^{i-1 \rightarrow i}$ \\
$R_{j-j+1}^{i}$ & $R_{j-1 \rightarrow j}^{i}$ \\
$C_{j}^{i-i+1}$ & $C_{j}^{i-i \rightarrow i}$ \\
\hline
\end{tabular}

If we call the time taken to execute a single task sequentially a "time-step", then figure 3 shows us that a minimum of six time-steps are required [in general, $2(N-L)$ time-steps will be required]. Also, six processing elements are required [2(N-L) in general].

Although, for a particular set of precedence constraints, the maximally paralkel task system is uniquely identifiable and gives us execution in a minimum number of time-steps, it is not the most efficient with respect to the number of processors utilized. In general, criteria which govern the choice of a parallel algorithm are:

1. execution time (i.e., the number of time-steps);

2. number of processing elements used;

3. memory requirement for each processing element;

4. programming simplicity.

The maximally parallel task system gives us an optimal parallel algorithm with respect to only the first of these criteria, viz., the execution time. Although it is possible, given a general set of precedence constraints, to arrive at a parallel algorithm which is optimal with respect to both execution time and number of processing elements used (this algorithm will in general not be unique), it need not be optimal with respect to the other two criteria. A decision based on all four criteria is very problem-

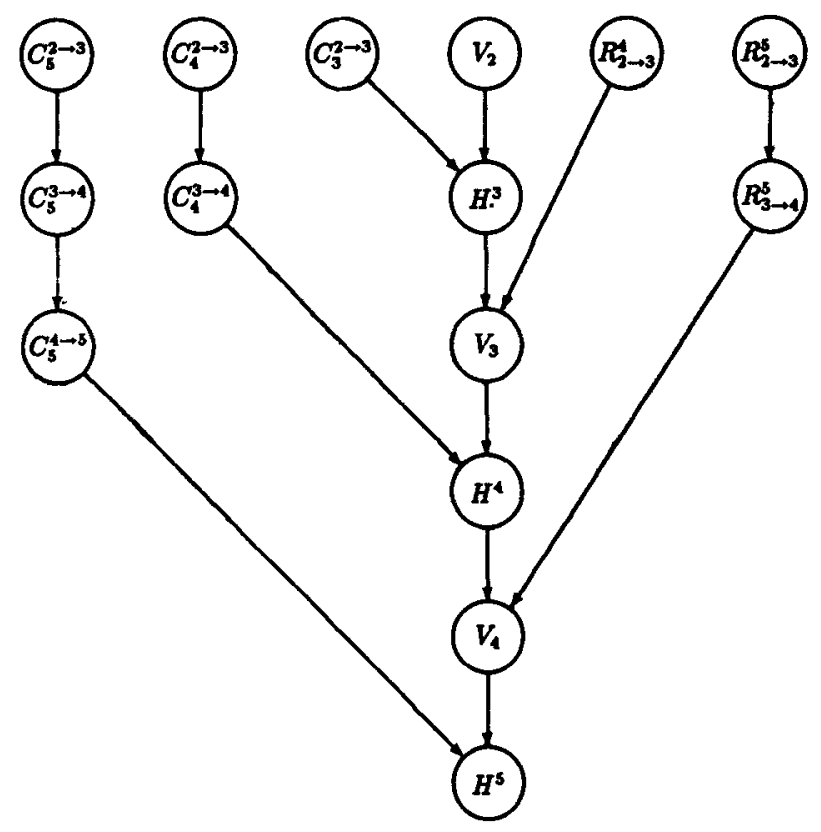

Figure 3. Maximally parallel task system. 


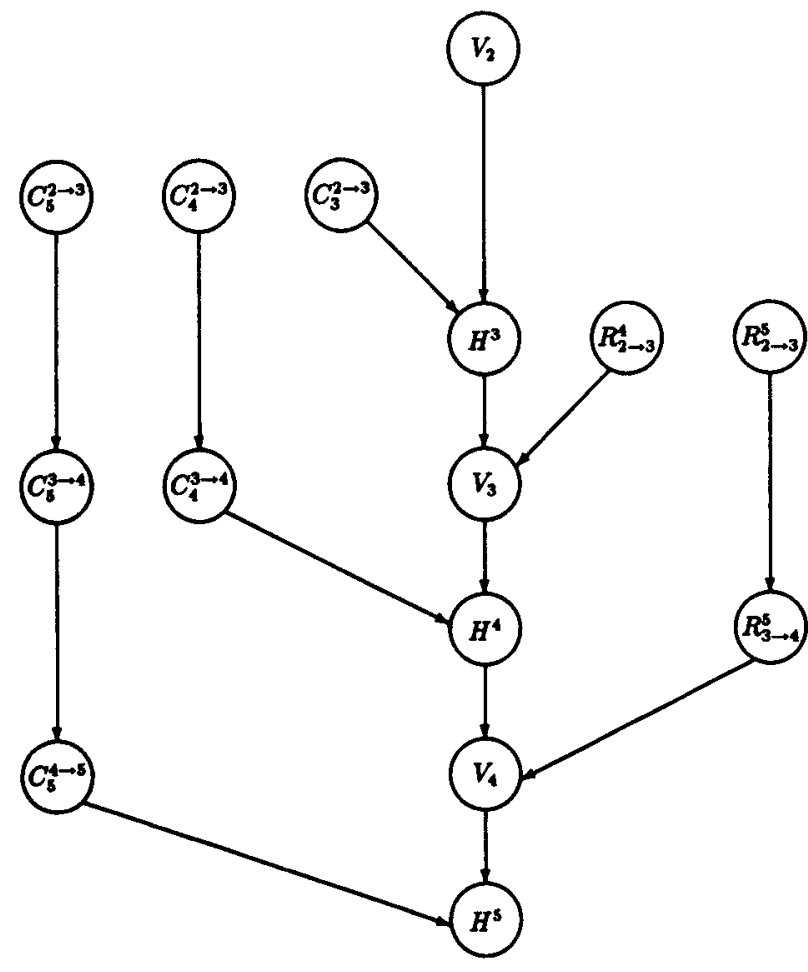

Figure 4. Task system for the parallel A2D-RLS algorithm.

dependent, and merely specifying a set of precedence constraints does not give us an answer.

Keeping these four criteria in mind, we have arrived at a parallel algorithm, the task system diagram of which is shown in figure 4 . Note that now, although the number of time-steps has increased to seven $[2(N-L)+1$ in general], the number of processing elements* has reduced relative to the maximally parallel task system, from six to three ( $N-L$ in general). Assuming that $N$ is large in comparison with unity, as is generally the case, we find that this task system is twice as efficient as the maximally parallel task system.

We now present a scheme for the implementation of the parallel task system introduced above. This scheme employs a linear array of processing elements, and is apropos for implementation on a distributed memory MIMD multiprocessor. An illustration of the scheme for a $7 \times 7$ image and a $3 \times 3$ non-causal window is given in figure 5 . Processing elements are represented by boxes containing the processing element number. Tasks listed horizontally all execute concurrently in a particular time-step. The column of tasks below a processing element represents the tasks executed by that processing element at various time-steps. Arrows between rows of tasks (i.e., between time-steps) show that the results of a task executed on one processing element are communicated to a neighbouring processing element, the direction of communication being given by the direction of the arrow. Thus, for example, in figure 5 , processing element 2 communicates the results of $R_{2 \rightarrow 3}^{4}$ to

\footnotetext{
* The number of processing elements required is the maximum overall time-steps of the number of tasks to be completed in a single time-step.
} 


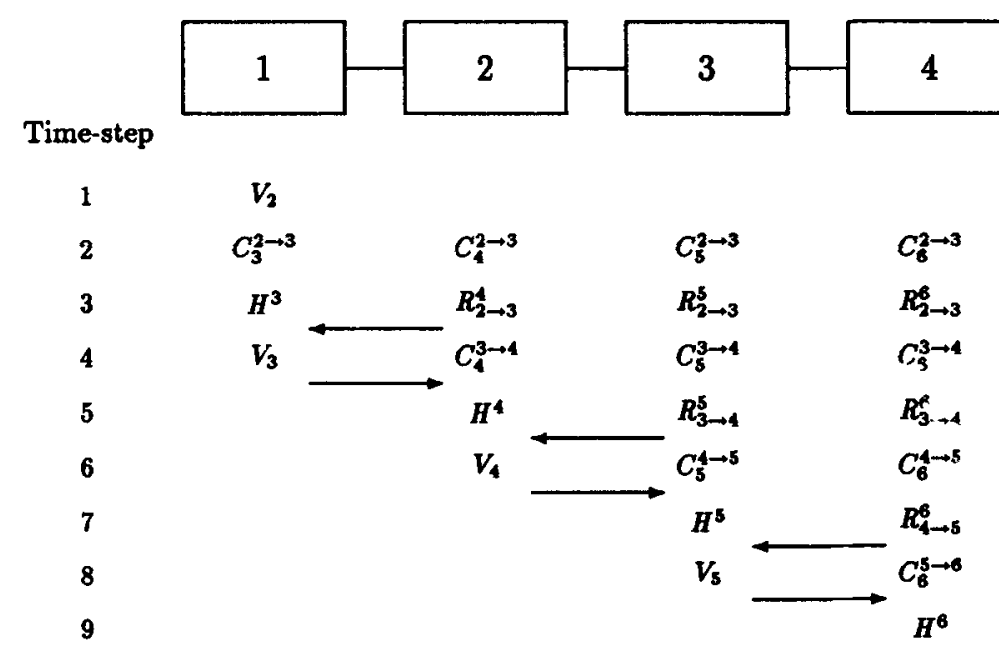

Figure 5. The parallel scheme for the A2D-RLS algorithm.

processor 1, which needs these data to commence execution of $V_{3}$. This communication has been represented by an arrow pointing to the left between time-steps 3 and 4 .

Several points are worthy of note in this figure. First, observe that there is only a single communication between two time-steps. This implies that it would also be possible to use a unibus architecture (figure 6 ) as is to be found on computers such as the S/NET developed at AT\&T Bell Laboratories. Second, note that a bidirectional communication link is required.

We have so far been purposely using the term processing elements, as opposed to physical processors. This is because, in general, we do not have $(N-L)$ processors available for solving our problem. Typically, the number of processors is much less - anywhere from 2 to 32 or even 64 processors. Hence, the scheme developed by us so far cannot directly be used on an actual multiprocessor. How then does one take into account the effect of these reduced number of processors? The answer is simple. Let us say we have only four processors available. The tasks assigned to processing elements $1,2,3$ and 4 get assigned to the corresponding processors. Now however, we assign the tasks of processing element 5 to processor 1 , those of processing element 6 to processor 2 and so on. Similarly, the tasks assigned to every $Z$ th processing element gets assigned to a single processor, where the number of processors is $Z$. Thus, the tasks assigned to processing element $k$ are assigned to processor $k \bmod (Z)$. Note, however, that now the result of the $H$ task computed by the last processor in the linear array is required by the first processor of the array for doing the next $V$ task. Hence, we require a ring topology, and not just a linear array for implementing our algorithm. One of the subtle points easily overlooked in the above discussion is the existence of an extra $V$ task on the first processor; for the four processor example, in addition to the set of tasks from processing element 5 , processor 1 will also be assigned one extra task viz., $V_{6}$. Also, one $V$ task will be missing for the last processor.

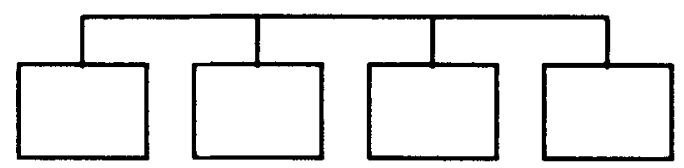

Figure 6. A unibus architecture. 
Again, for the four processor example, all tasks from processing element 8 would be assigned to processor 4 except the $V$ task assigned to that processing element.

What happens if the number of processing elements is not an exact multiple of the number of processors? Again, the answer is not difficult - we pad the image with extra rows and columns until it is indeed a multiple, but we stop the program after having completed the last relevant update.

Next we consider the effect of including the initialization of $\alpha$ and $\beta$ to the latest value of $\hat{\theta}$. Clearly, if we were to wait for the very latest value of $\hat{\theta}$ to be conveyed by the preceding processor before beginning work on the $C$ and $R$ tasks, the parallelism in the algorithm would be completely lost; we would have a purely sequential algorithm at our hands. A compromise can be achieved if we do not use the very latest value of $\hat{\theta}$, but the last computed value of $\hat{\theta}$ residing on the current processor. Thus, as the number of processors increases, the age of the $\hat{\theta}$ being used for initializing $\alpha$ or $\beta$ also increases. Thus, we can expect a degradation in the performance of the algorithm as the number of processors increases, and we have found that this indeed happens (unfortunately). However, all is not lost-we have gained by parallelizing our algorithm and so reducing the execution time.

As concerns the re-initialization, it is evident that it can very easily be implemented merely by comparing $p(\cdot, \cdot)$ with a threshold immediately after completing a $V$ or $H$ task on any of the processors, and setting its value to the starting value if it turns out that $p$ is too small.

At this juncture, we choose to substantiate our claim that an assumption that all tasks have equal computational complexity would not degrade performance. In practice, the size of the image would be quite large, and so the number of $R$ and $C$ tasks done by a typical processor would far outnumber the $V$ and $H$ tasks done by that processor. Since the computational complexity of a $V$ or $H$ task is in reality less than that of a $C$ or $R$ task, our assumption of equal computational complexities would not seriously affect performance.

Let us now analyse the data requirements for each processor. For this, we need consider only the $R$ and $C$ tasks, since only these tasks require data from the image. Each $R^{i}$ task requires data from a non-causal window centred at a point on the $i$ th row of the image. Similarly, each $C_{j}$ task requires data from a non-causal window centred at a point on the $j$ th column of the image. We note that if $R_{2 \rightarrow 3}^{k}$ is scheduled for execution on a particular processor, that processor will also do all other $R$ tasks having the same superscript. A similar statement can be arrived at for $C$ tasks. Thus, a processor will require $L$ rows of data from the image for each $k$, where $R_{x \rightarrow x+1}^{k}$ is scheduled for execution on that processor. Similarly, $L$ columns of data from the image will be required for each $l$, where $C_{l}^{y \rightarrow y+1}$ is scheduled for execution on that processor. Also, note that not all these sets of $L$ rows and $L$ columns need be stored - we can store all data values from row 1 or column 1 up to the diagonal of the image, along the corresponding column or row respectively. This is best illustrated by the diagram in figure 7 . Note that although it is not explicitly shown in this figure, there will be substantial overlapping of data between processors.

\section{Implementation results}

We have implemented our parallel algorithm using a parallel computer simulator and performance monitor called ASTRO (Dittia \& Desai 1989). The machine used for 


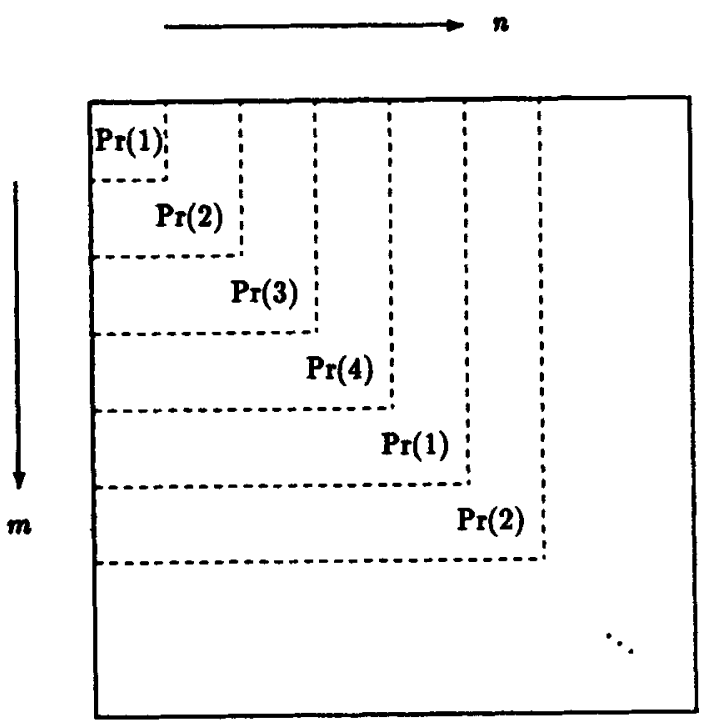

Figure 7. Data requirement for processors.

the simulation was a personal computer. ASTRO simulates a distributed memory multiprocessor with a loosely synchronous concurrent computing environment. After executing the parallel program, it directly provides the speed-up, efficiency and fractional communication overhead. These results do not take into account load balancing, and hence are not accurate estimates of the actual performance that would be obtained on a real parallel computer. However, they give a good means of analysing the behaviour that the speed-up, efficiency and fractional communication overhead would exhibit when parameters such as the number of processors are changed.

For the purposes of illustration, we have used a synthetic $256 \times 256$ AR image generated using a $3 \times 3$ causal window, the parameter vector of which was:

$$
\theta^{T}=\begin{array}{ccc}
{[0.9025} & -0.1853 & 0.95 \\
-0.1825 & 0.03803 & -0.195 \\
0.95 & -0.195 & \text { null }]
\end{array}
$$

The noise term $v(m, n)$ was chosen to be white gaussian with zero mean and variance 0.0731 , which resulted in a signal variance of 6 .

The first three plots (figures 8-10) we have obtained relate to the variation of the parallel computer performance, while the other two (figures $11 \& 12$ ) give us an idea of the algorithm's performance.

The first graph (figure 8) is a plot of the speed-up as a function of the number of processors. This plot reveals a surprising fact - the speed-up increases almost linearly with the number of processors. This means that we can use a large number of processors efficiently, a fact that is substantiated by the second plot of efficiency versus the number of processors (figure 9). It is seen that efficiency remains nearly constant at $67 \%$. Another consequence of a linearly increasing speed-up is that the time spent on communication does not rise rapidly if we increase the number of processors; in fact, the rise in communication time is proportional to the rise in total computation time, a point that is clearly brought out by the third graph (figure 10), which is a plot of the fractional communication overhead versus the number of processors. It is seen 


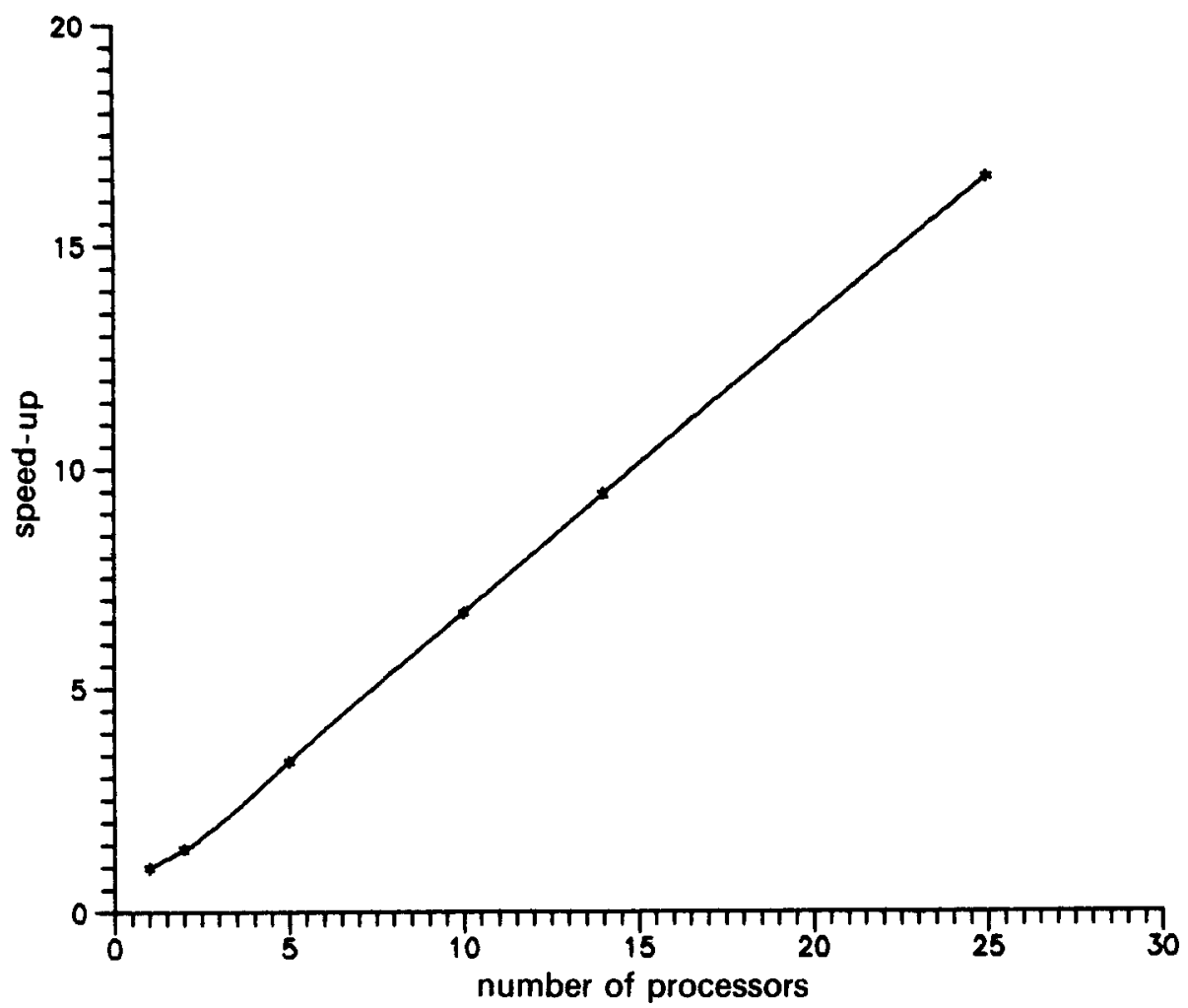

Figure 8. Speed-up vs. number of processors (window size $=3$ ).

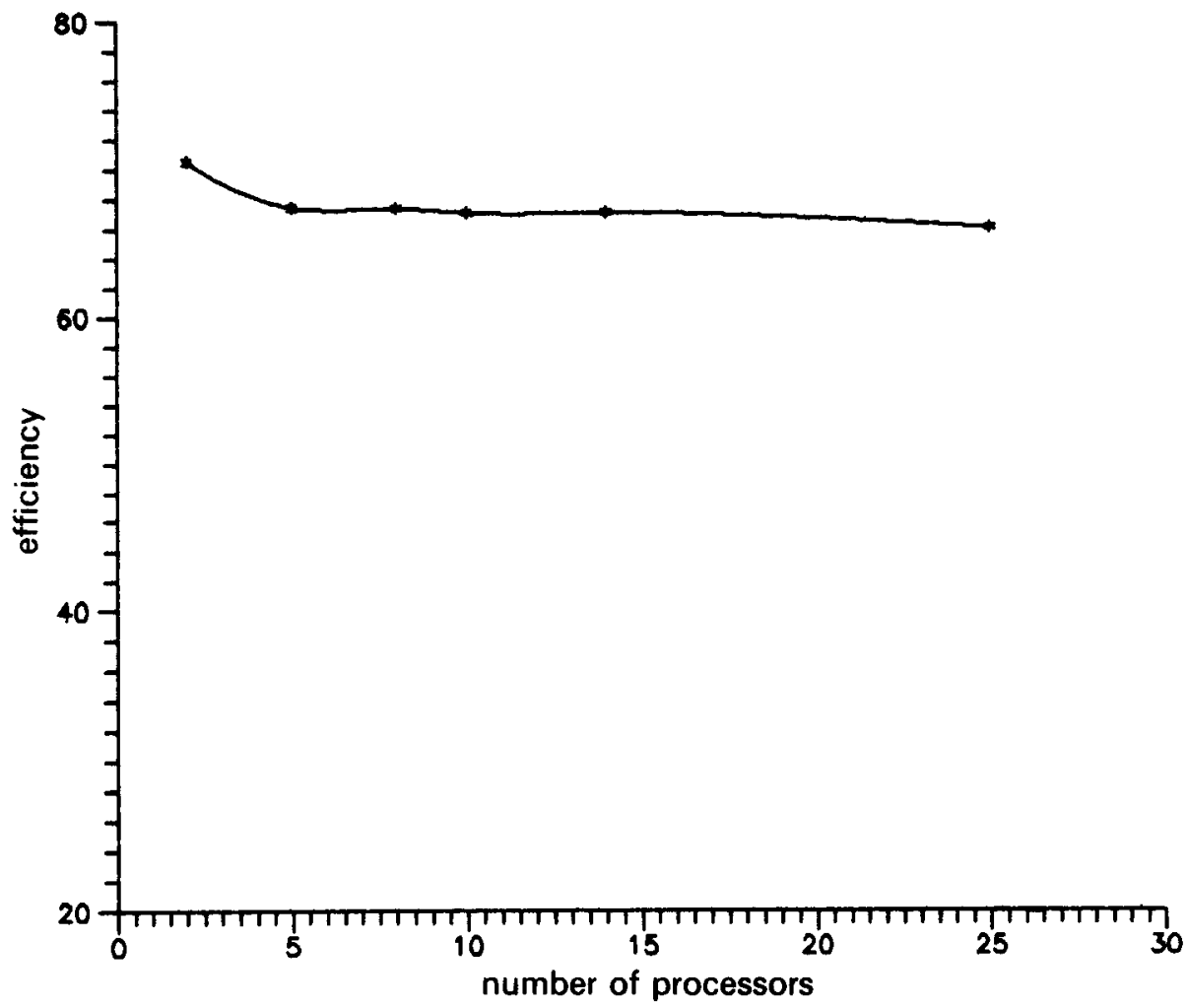

Figure 9. Efficiency vs. number of processors (window size = 3). 


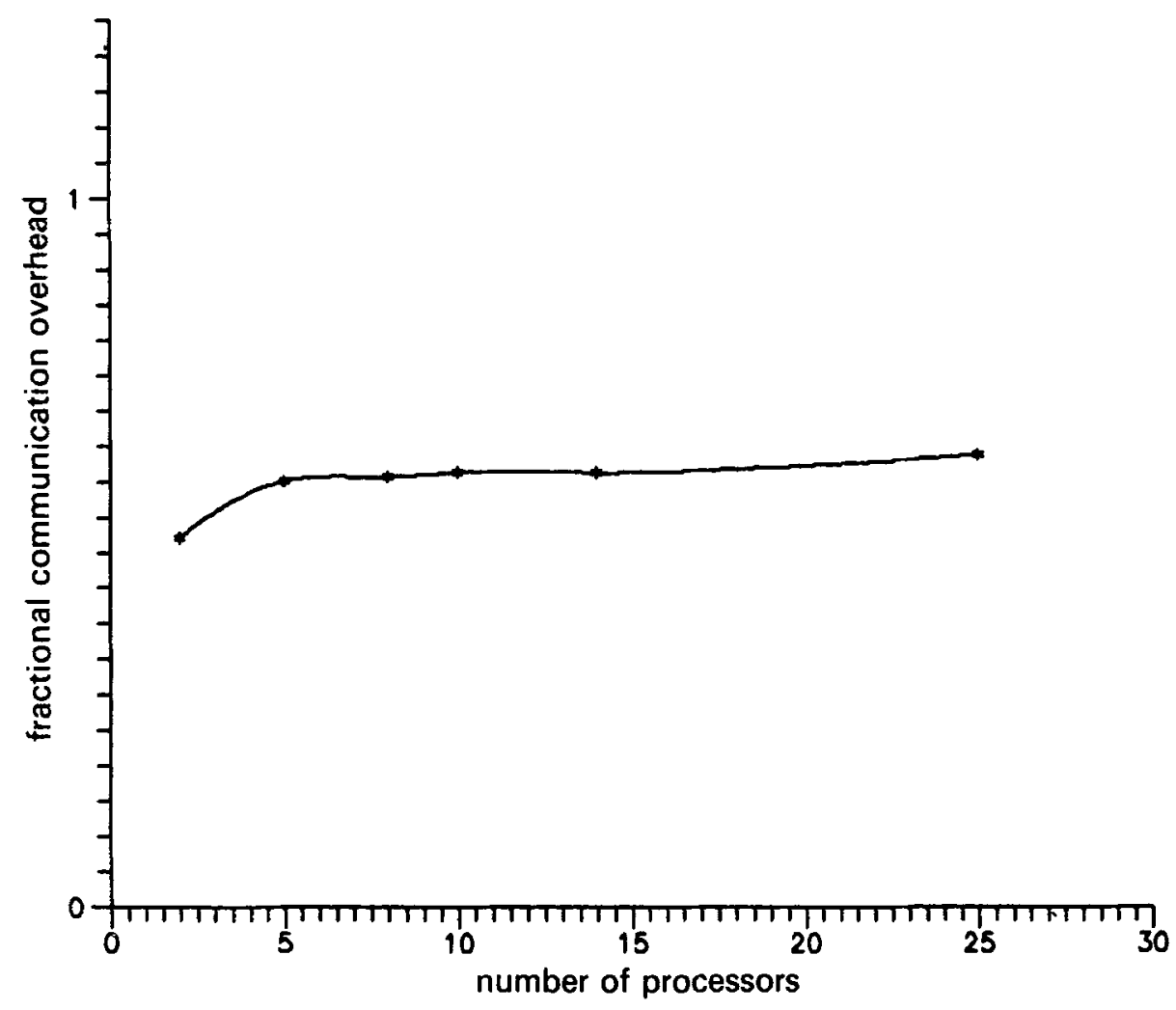

Figure 10. Fractional communication overhead vs. number of processors (window size $=3$ ).

from this plot that the variation is very small, i.e. the fraction of the total run time spent on communication remains very nearly constant.

Thus it seems we can utilize a large number of processors very efficiently for implementing our algorithm. But there is a catch. Recall that we are using the latest value of $\hat{\theta}$ present on the current processor to initialize $\alpha$ and $\beta$, rather than the very latest value of $\hat{\theta}$ that has been computed. This means, as stated earlier, that for more processors, we are using older values of $\hat{\theta}$ for the initialization. Hence, we expect a degradation in the convergence rate of the algorithm as the number of processors increases. The fourth graph (figure 11 ) is a plot of the parameter $\hat{\theta}(0,2)$ (remember that our experiments used a causal model) as the algorithm proceeds, for two parallel computers, one having five processors and the other having ten. We see that the rate of convergence for the latter is much lower, so much so that the 508 updates are insufficient to converge to the desired value. This reflects as an increased error in the final parameter vector $\theta$. This observation is substantiated by the fifth graph (figure 12), which is a plot of the percentage error in the computed value of $\theta$ versus the number of processors. The degradation in the performance of the algorithm as the number of processors increases is evident. A reasonable number of processors for a $256 \times 256$ image is seen to be around ten, where we obtain an error of $20 \%$. Of course, for larger images, there will be more iterations and consequently more time to converge; hence, we can use more processors and yet attain the same error in the estimated parameters. 


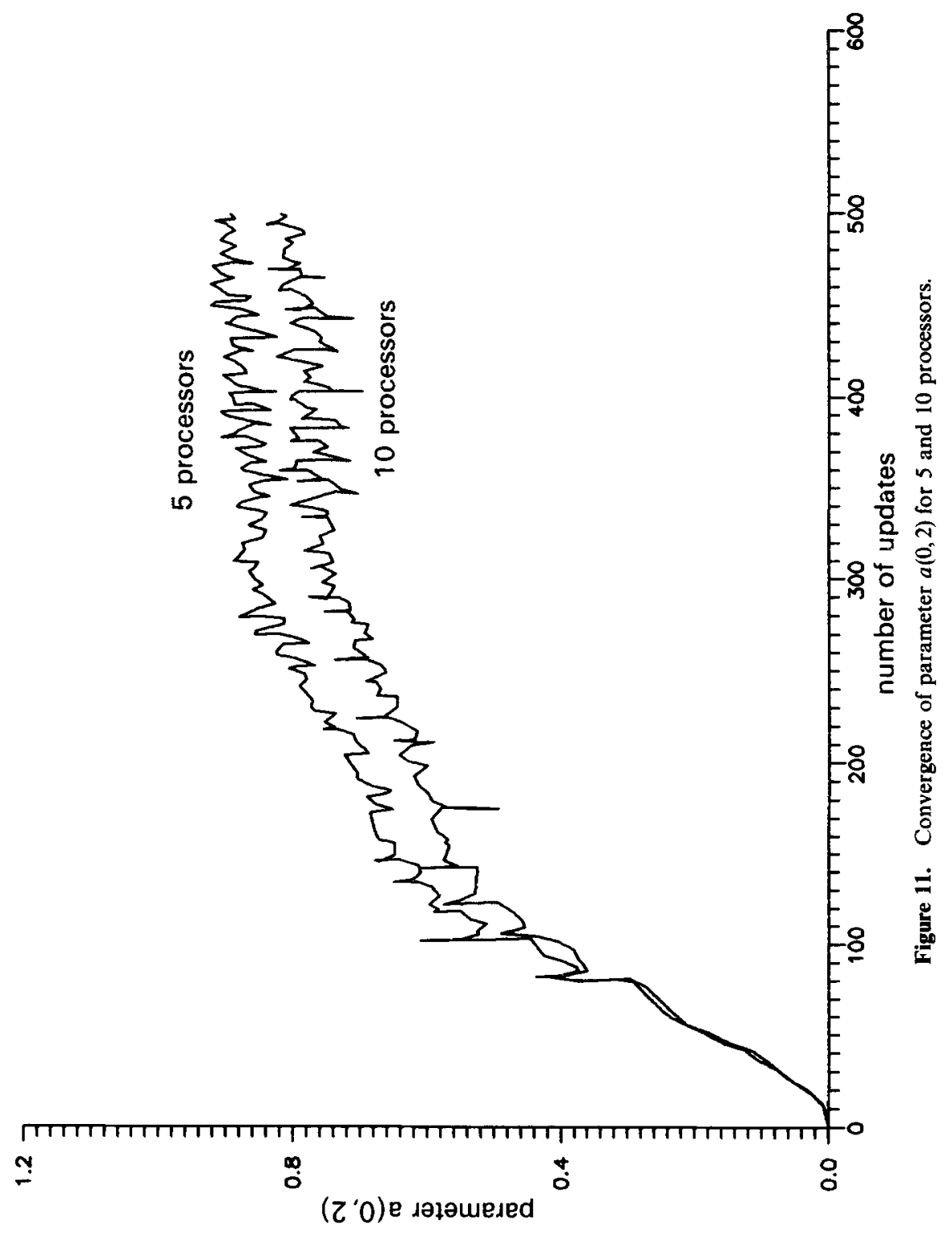




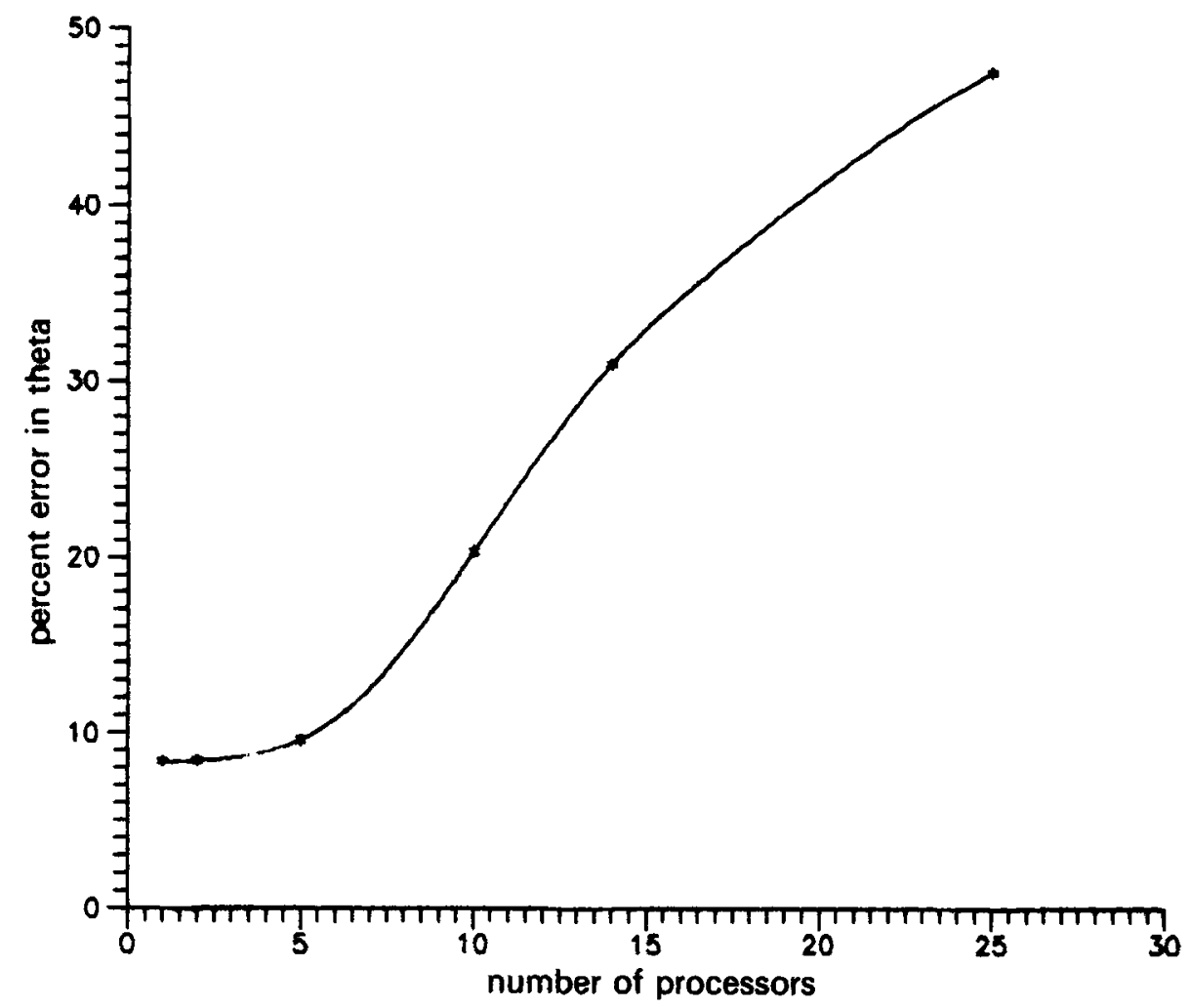

Figne 12. Percentage error in parameters vs. number of processors (window size $=3$ ).

\subsection{Image estimation}

To test the utility of our algorithm for the image estimation problem, we applied it to the estimation of the girl image (shown in figure 13) from a noisy image. Two cases of the noisy image were considered; one with a signal-to-noise ratio (SNR) of $12 \mathrm{~dB}$, and the other with an SNR of $3 \mathrm{~dB}$. Also for purposes of comparison, we also applied the 2D-LMS algorithm (Hadhoud \& Thomas 1988), and the F2D-RLS algorithm (Boutalis et al 1989).

The noisy image and the estimated images, for the $3 \mathrm{~dB}$ SNR case, are depicted in figures $14-17$. It is to be noted that the algorithms were applied using a $3 \times 3(L=3)$ noncausal window. Moreover the images were estimated adaptively, i.e. pixel $(i, j)$

Table 6. SNR improvements in estimated images.

\begin{tabular}{lccc}
\hline & $\begin{array}{c}\text { Input SNR } \\
\text { (dB) }\end{array}$ & $\begin{array}{c}\text { Output SNR } \\
\text { (dB) }\end{array}$ & $\begin{array}{c}\text { SNR improvement } \\
\text { (dB) }\end{array}$ \\
\hline A2D-RLS & 12.49 & 15.26 & 2.77 \\
2D-LMS & 12.49 & 14.78 & 2.29 \\
Fast-RLS & 12.49 & 12.14 & -0.35 \\
A2D-RLS & 3.0 & 10.36 & 7.36 \\
2D-LMS & 3.0 & 10.34 & 7.34 \\
Fast-RLS & 3.0 & 8.5 & 5.5 \\
\hline
\end{tabular}



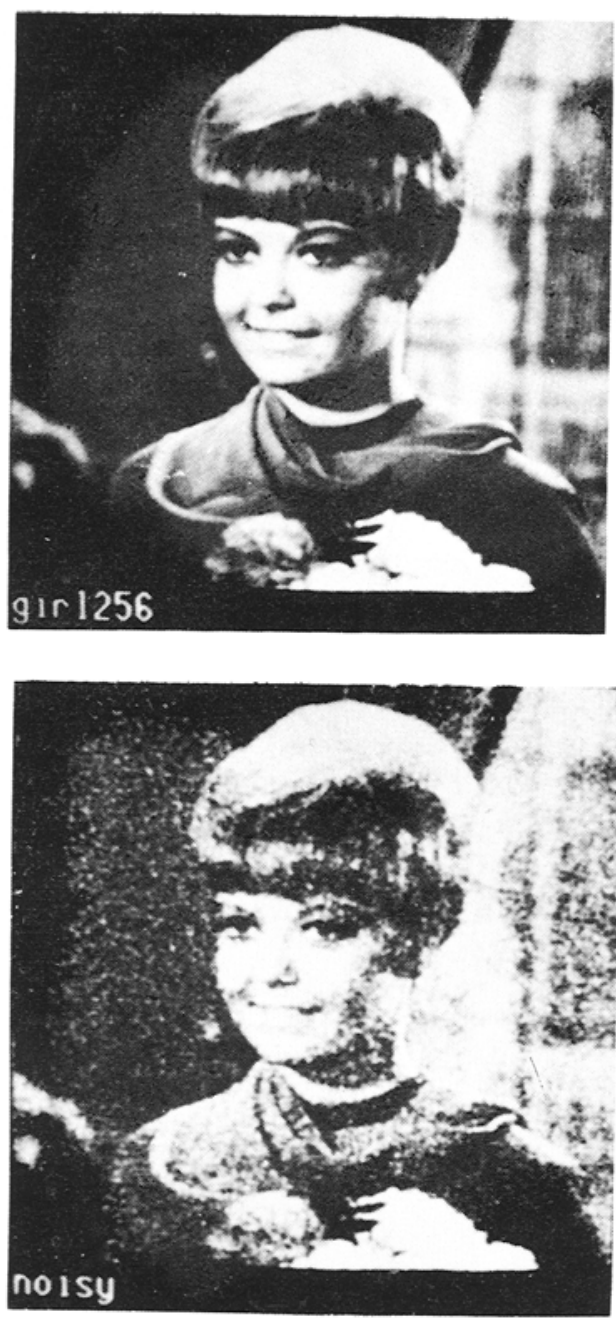

Figure 14. Noisy image (SNR $3 \mathrm{~dB}$ ).

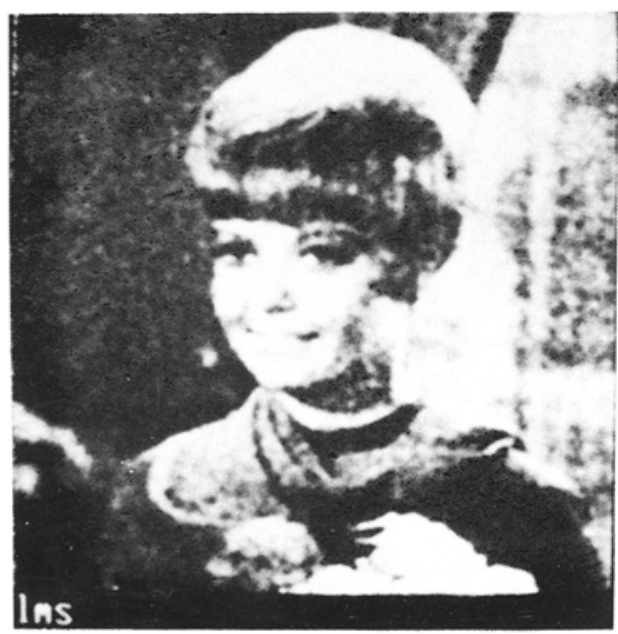

Figure 16. Estimated image using 2D-LMS.
Figure 13. Original image.

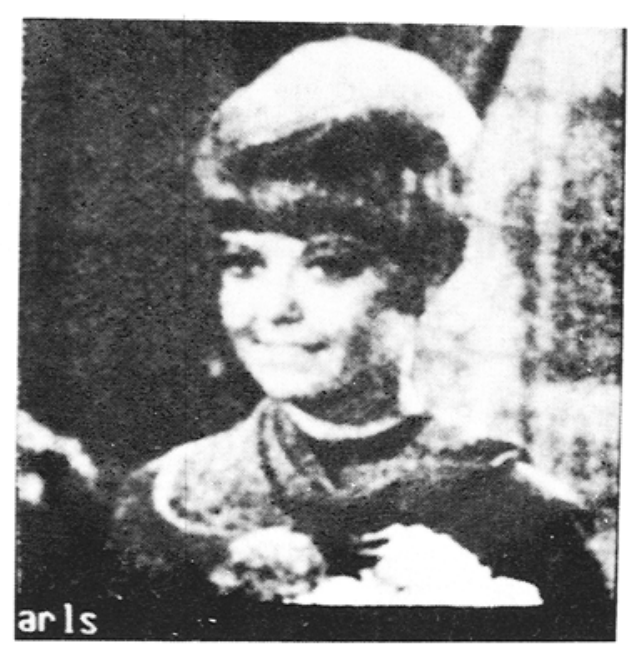

Figure 15. Estimated image using A2D-RLS.

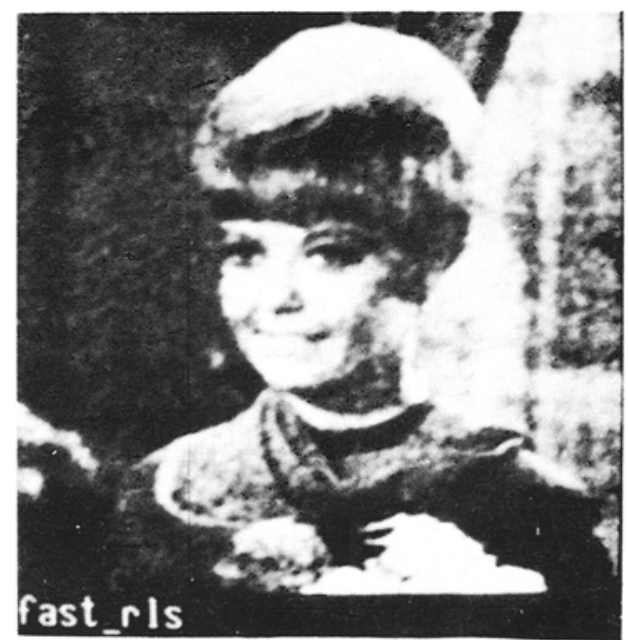

Figure 17. Estimated image using F2D-RLS. 
was estimated using the current estimate of $\theta$, nameiy $\theta(i, j)$. The SNR improvements in the estimated images are listed in table 6.

In both the cases A2D-RLS gave the best output SNR.

\section{Conclusions}

A parallel scheme for the implementation of the A2D-RLS algorithm was developed, and was implemented using a parallel computer simulator. The performance results show that although we have achieved a high degree of parallelism, we cannot go on increasing the number of processors, as this results in a degradation in the estimated parameters. If we fix the percentage error in the estimated parameters to a certain allowable maximum, we arrive at an upper bound on the number of processors we can use. However, this upper bound would vary depending upon the size of the image and upon the window size. A larger image would facilitate the use of more processors. The speed-up was seen to increase almost linearly with the number of processors, implying a very small communication overhead.

Both the 2D-RLS and the A2D-RLS algorithms exhibit the same degree of parallelism. However, the latter remains a much faster approach to 2-D parameter estimation; hence our concentration on it. With appropriate modifications our parallelization scheme can easily be extended to the 2D-RLS algorithm.

Finally, in the image estimation context, comparing speed, output SNR, and general quality of the estimated image, we feel that parallel A2D-RLS offers a good alternative to image estimation algorithms.

\section{References}

Boutalis Y S, Kollias S D, Carayannis G 1989 A fast multichannel approach to adaptive image estimation. IEEE Trans. Acoust.. Speech Signal Process. ASSP-37: 1090-1098

Chan P, Lim J S 1985 One-dimensional processing for adaptive image restoration. IEEE Trans. Acoust. Speech Signal Process. ASSP-33: 117-126

Desai U B, Kumar P S 1989 An approximate 2D-RLS algorithm with application to image estimation. Indo-US Workshop on Spectral Analysis in One or Two Dimensions, New Delhi

Dittia Z D. Desai U B 1989 ASTRO - A Pseudo Compiler for Parallel Computer Simulation on a Personal Computer. Technical Report, Department of Electrical Engineering, Indian Institute of T echnology, Bombay

Fox G, Johnson M, Lyzenga G, Otto S. Salmon J, Walker D 1988 Solving problems on concurrent processors (Englewood Cliffs, NJ: Prentice Hall)

Hadhoud M M, Thomas D W 1988 The two-dimensional adaptive LMS (TDLMS) algorithm. IEEE Trans. Circuits Syst. 35: 485-494

Haykin S 1986 Adaptive filter theory (Englewood Cliffs, NJ: Prentice Hall)

Kalouptsidis N, Theodoridis S 1989 Concurrent algorithms for a class of 1-D and 2-D Wiener filters with symmetrical impulse response. IEEE Trans. Acoust. Speech Signal Process. ASSP-37: 1780-1782

Kumar S P, Kowalik J S 1986 Triangularization of a positive definite matrix on a parallel computer. $J$. Parallel Distributed Computing 3: 450-460

Lord R E, Kowalik J S, Kumar S P 1980 Solving Linear Algebraic Equations on a MIMD Computer. Proc. Int. Conf. on Parallel Processing, IEEE

Takalp A, Kaufman H, Woods J 1985 Fast recursive estimation of the parameters of space varying AR images. IEEE Trans. Acoust. Speech Signal Process. ASSP-33: 469-471

Widrow B, Stearns S D 1985 Adaptive signal processing. (Englewood Cliffs, NJ: Prentice Hall) 